\title{
Pacing during an ultramarathon running event in hilly terrain
}

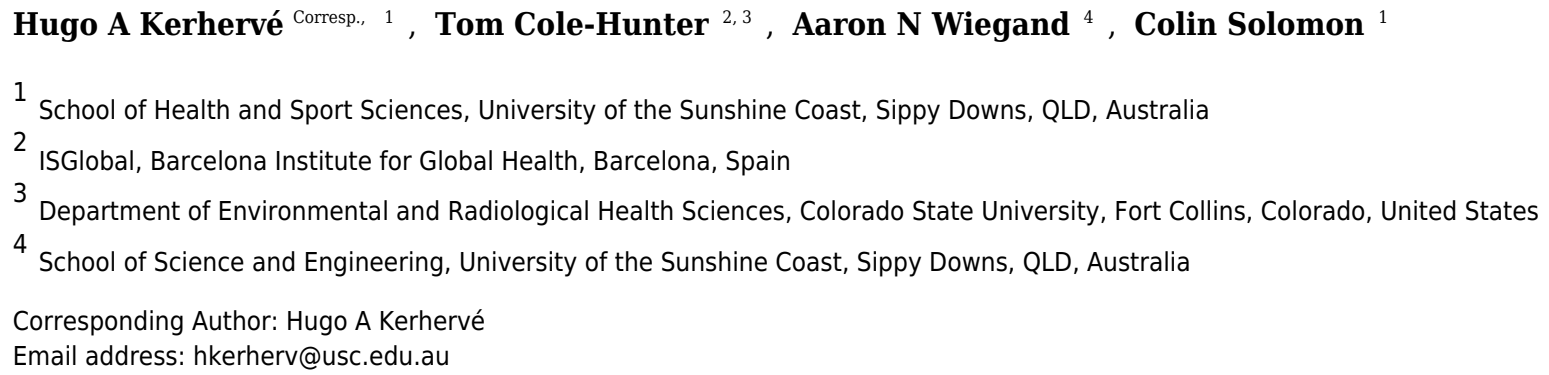

Purpose. The dynamics of speed selection as a function of distance, or pacing, are used in recreational, competitive, and scientific research situations as an indirect measure of the psycho-physiological status of an individual. The purpose of this study was to determine pacing on level, uphill and downhill sections of participants in a long (>80km) ultramarathon performed on trails in hilly terrain. Methods. Fifteen ultramarathon runners competed in a $\sim 173 \mathrm{~km}$ event (five finished at $\sim 103 \mathrm{~km}$ ) carrying a Global-Positioning System (GPS) device. Using the GPS data, we determined the speed, relative to average total speed, in level (LEV), uphill (UH) and downhill (DH) gradient categories as a function of total distance, as well as the correlation between overall performance and speed variability, speed loss, and total time stopped. Results. There were no significant differences in normality, variances or means in the relative speed in $173-\mathrm{km}$ and $103-\mathrm{km}$ participants. Relative speed decreased in LEV, UH and DH. The main component of speed loss occurred between $5 \%$ and $50 \%$ of the event distance in LEV, and between $5 \%$ and $95 \%$ in $\mathrm{UH}$ and $\mathrm{DH}$. There were no significant correlations between overall performance and speed loss, the variability of speed, or total time stopped. Conclusions. Positive pacing was observed at all gradients, with the main component of speed loss occurring earlier (mixed pacing) in LEV compared to UH and DH. A speed reserve (increased speed in the last section) was observed in LEV and UH. The decrease in speed and variability of speed were more important in LEV and DH than in UH. The absence of a significant correlation between overall performance and descriptors of pacing is novel and indicates that pacing in ultramarathons in trails and hilly terrain differs to other types of running events. 
$1 \quad$ Pacing during an ultramarathon running event in hilly terrain

2

Hugo A. Kerhervé ${ }^{1}$, Tom Cole-Hunter ${ }^{2,3}$, Aaron N. Wiegand ${ }^{4}$, Colin Solomon ${ }^{1}$

$3{ }^{1}$ School of Health and Sport Sciences, University of the Sunshine Coast, QLD Australia ${ }^{2}$

4 ISGlobal, Barcelona Institute for Global Health, Barcelona, Spain

$5{ }^{3}$ Department of Environmental and Radiological Health Sciences, Colorado State University,

6 Fort Collins, Colorado, USA

$7{ }^{4}$ School of Science and Engineering, University of the Sunshine Coast, QLD Australia

8

9 Submission type:

10 Original research article

11 Preferred running head:

12 Pacing during a hilly ultramarathon

13 Word count:

14 Abstract: $294 \mathrm{w}$

15 Text only: $4083 \mathrm{w}$

16 Supplemental files:

17 https://dx.doi.org/10.6084/m9.figshare.3369790.v1 


\section{Abstract}

19 Purpose. The dynamics of speed selection as a function of distance, or pacing, are used in recreational, competitive, and scientific research situations as an indirect measure of the psychophysiological status of an individual. The purpose of this study was to determine pacing on level, uphill and downhill sections of participants in a long $(\sim 173 \mathrm{~km})$ ultramarathon performed on trails in hilly terrain.

Methods. Fifteen ultramarathon runners competed in a $\sim 173 \mathrm{~km}$ event (five finished at $\sim 103 \mathrm{~km}$ ) carrying a Global-Positioning System (GPS) device. Using the GPS data, we determined the speed, relative to average total speed, in level (LEV), uphill (UH) and downhill (DH) gradient categories as a function of total distance, as well as the correlation between overall performance and speed variability, speed loss, and total time stopped.

Results. There were no significant differences in normality, variances or means in the relative speed in 173-km and 103-km participants. Relative speed decreased in LEV, UH and DH. The main component of speed loss occurred between $5 \%$ and $50 \%$ of the event distance in LEV, and between $5 \%$ and $95 \%$ in $\mathrm{UH}$ and $\mathrm{DH}$. There were no significant correlations between overall performance and speed loss, the variability of speed, or total time stopped.

Conclusions. Positive pacing was observed at all gradients, with the main component of speed loss occurring earlier (mixed pacing) in LEV compared to UH and DH. A speed reserve (increased speed in the last section) was observed in LEV and UH. The decrease in speed and variability of speed were more important in LEV and DH than in UH. The absence of a significant correlation between overall performance and descriptors of pacing is novel and indicates that pacing in ultramarathons in trails and hilly terrain differs to other types of running events.

\section{Keywords}

41 Running; Performance; Global Positioning System; Elevation Gain and Loss; Digital Elevation 42 Model 
43 Introduction

44 Paragraph \#1. The dynamics of speed during self-paced locomotor exercise, or pacing, are used in recreational, competitive and scientific settings as an indicator of exercise intensity (Abbiss \& Laursen 2008), and fatigue (Knicker et al. 2011). Three general types of pacing (negative, even, positive) are commonly identified in the analysis of running performance, using the direction of the changes in time per $\mathrm{km}$ or speed, as a function of distance or exercise duration. Events longer than the marathon (ultramarathon, UM) are increasingly popular in recreational and competitive settings and are increasing being used in scientific research. However, there is a relative paucity of systematic descriptions of pacing in long $(>80 \mathrm{~km}) \mathrm{UM}$, especially those performed on trails and in hilly or mountainous terrain, which could be due to the difficulties associated with the monitoring of individual participants in remote areas and over long durations.

Paragraph \#2. Positive pacing (decreasing speed) and a subset of positive pacing including a final end-spurt referred to as parabolic pacing (Abbiss \& Laursen 2008), were observed in UM, such as during a short $(45 \mathrm{~km}$ ) trail UM in recreational runners (Angus \& Waterhouse 2011), a $100 \mathrm{~km}$ event on a level, multi-loop course in elite runners (Lambert et al. 2004), a $105 \mathrm{~km}$ mountain trail UM in competitive runners (Kerhervé et al. 2015), and a $161 \mathrm{~km}$ mountain trail UM in the five fastest runners over a 28 year period (Hoffmann 2014). Positive pacing has also been observed in other forms of ultra-endurance exercise, such as a $24 \mathrm{~h}$ treadmill run (Gimenez et al. 2013), or during an ultra-endurance triathlon event consisting of ten consecutive Ironman distance triathlons (10 x $3.8 \mathrm{~km}$ swimming, $180 \mathrm{~km}$ cycling, $42 \mathrm{~km}$ running) in 10 days (Herbst et al. 2011). Pacing is also characterised by the magnitude of speed loss and the variability of speed (using the coefficient of variation of speed), which were found to be lower in faster compared to slower participants during UM running events (Lambert et al., 2004;Hofmann, 2014), in agreement with what has been observed in events up to the marathon distance (Ely et al. 2008; Haney \& Mercer 2011).

Paragraph \#3. In contrast to these findings, we measured in a previous study on trained UM runners a higher magnitude of speed loss in faster compared to slower runners, no significant relationship between the variability of speed and performance level, and a novel significant negative relationship between the total time stopped and performance level, in a long mountain UM (Kerhervé et al. 2015). Additionally, speed on level, uphill and downhill sections increased in 
73 the last $10 \%$ of the event. These results indicate that pacing may have been regulated 74 conservatively in anticipation of topographic difficulties, and that faster runners paced less conservatively than slower runners. Overall, the literature specific to UM and ultra-endurance exercise has highlighted the protective nature of fatigue in situations where the physical integrity of participants could be compromised, such as greater neuromuscular (Millet et al. 2011b) or biomechanical alterations (Morin et al. 2011b) in events with large elevation gain and loss compared to UM events on level ground (Martin et al. 2010; Morin et al. 2011a).

Paragraph \#4. Therefore, the aim of this study was to record the individual pacing of participants in a long trail UM in hilly terrain, in order i) to measure the dynamics of speed selection as a function of terrain (level, uphill and downhill sections), and ii) to further investigate the relationship between performance and pacing characteristics (speed loss, speed variability, total time stopped). Speed was expected to decrease in all participants and at all gradients as a function of the distance and duration of the event.

\section{Methods}

Paragraph \#5. This study was approved by the university research ethics committee (Queensland University of Technology, project 0900001233). The study participants were recruited using advertisements on a specialised forum and researchers networks, from individuals already registered to compete in the Great North Walk 100s (NSW, Australia), a long ( 173 km) and hilly UM running event including 6 checkpoints and a total elevation gain and loss of approximately $3,000 \mathrm{~m}$. This event offered the opportunity to receive an official classification and time for participants failing to complete the entire event if they reached the $4^{\text {th }}$ checkpoint at $\sim 103 \mathrm{~km}$, which we included in the study. Both distances are presented in this article as $173-\mathrm{km}$ and $103-$ $\mathrm{km}$. After providing written informed consent, 19 participants were equipped with a commerciallyavailable Global Positioning System (GPS) device (BT-Q1000, Qstarz International, Taiwan) fitted to their clothing or pack.

Paragraph \#6. The GPS devices used in this study were selected for their light weight ( $\sim 100 \mathrm{~g}$ including the battery) and long battery life (tested to record for more than 40 continuous hours at a sampling rate of $0.2 \mathrm{~Hz}$ ). The accuracy of measures of geographical position and speed were tested following published procedures (Townshend et al. 2008). Positional accuracy was found to 
102 be within the range provided by the manufacturer (100\% of measures within $3 \mathrm{~m}$ of a known 103 geodetic survey landmark, $84.5 \%$ of observations measured within $2 \mathrm{~m}$, and had a mean distance 104 of $1.57 \pm 0.43 \mathrm{~m}$ ). The calculated velocity (see following section) was found to be in excellent 105 agreement $(95 \%$ limits of agreement $=0.24 \pm 0.12$, typical error expressed as a $\%$ of $\mathrm{CV}=1.2$, 106 standardized error of the estimate $=0.03 \mathrm{~km} \cdot \mathrm{h}^{-1}$ ) and perfectly correlated to speed determined 107 using chronometry over a known distance $(100 \mathrm{~m})$ over 50 trials $(\mathrm{r}=1.00, \mathrm{p}<0.001)$.

108 Distance and Speed

109 Paragraph \#7. The distance between points at the surface of a sphere can be calculated using 110 simple spherical trigonometry. However, the earth is not a sphere, but an oblate spheroid akin to 111 an ellipsoid with the following dimensions: equatorial radius $\approx 6,378.13 \mathrm{~km}$, polar radius $\approx$ $1126,356.752314245 \mathrm{~km}$ and flattening $f \approx 1 / 298.257223563$ (Defense Mapping Agency 1990).

113 The calculation of point to point distances at the surface of an ellipsoid can be improved compared 114 to spherical trigonometry using the inverse Vincenty formulae (Vincenty 1975). The point-to-point 115 distances were obtained from an internet-based utility (GPS Visualizer; www.gpsvisualizer.com) 116 using geographical positions (latitude and longitude). We found the distances obtained were in 117 exact agreement $(\mathrm{r}=1.00, \mathrm{p}<0.001)$ with our preliminary measures of point-to-point distances 118 using the Vincenty formulae performed on 10 data sets. Therefore, we used the internet-based 119 calculations of the formulae as a simple and generalisable procedure to obtain point-to-point 120 distances.

121 Paragraph \#8. Point-to-point speed was subsequently calculated using the ratio of point-to-point 122 distances and time (one data point every 5 s) between each datum. Preliminary calculations 123 revealed that GPS devices did not discriminate for speeds slower than $1 \mathrm{~km} \cdot \mathrm{h}^{-1}\left(0.28 \mathrm{~m} \cdot \mathrm{s}^{-1}\right.$ 124 or $1.39 \mathrm{~m}$ in $5 \mathrm{~s}$ ) based on the typical error in speed in a static position (drift, when a device will 125 record speed values due to the non geo-synchronous nature of the constellation of satellites). At 126 the other end of the speed spectrum, it was considered that speeds higher than $20 \mathrm{~km} \cdot \mathrm{h}^{-1}(5.56$ $127 \mathrm{~m} \cdot \mathrm{s}^{-1}$ or $27.8 \mathrm{~m}$ in $5 \mathrm{~s}$ ) were not expected during a long UM and originated in signal jamming 128 (which can occur due to the signal from a satellite being too weak which forces the ground based 129 receiver to pair to another satellite). These erroneous distance and speed data were assigned a value 130 of zero, and all speed values were then smoothed in order to further increase the signal-to-noise 
131 ratio. For smoothing, a 9-pt weighted average was graphically compared with 3-pt and 15-pt

132 weighted averages and considered satisfactory as it provided a balanced sensitivity to individual 133 observations for slow and high speeds. This procedure limited the effect of signal drift and 134 jamming (higher distance and speed due to erroneous values). These procedures were sensitive to 135 periods of zero speed values, which corresponded to the location, via expected relative distances, 136 of checkpoints in the race.

\section{Elevation changes}

138 Paragraph \#9. We used previously published procedures (Kerhervé et al. 2015) to alleviate the 139 inaccuracies in GPS-based elevation (Townshend et al. 2008). The online utility (GPS Visualizer; 140 gpsvisualizer.com) was used to match the recorded geographical positions (determined by latitude 141 and longitude) to a DEM elevation datum from the National Aeronautics and Space Administration 142 (NASA) Shuttle Radar Topography Mission (SRTM) database. As the resolution of DEM is 143 relatively low for the study of human locomotion (90 m, only the SRTM3 was available where the current study was conducted), the procedure can create a series of steps when increasing or decreasing elevation every time a contour line is crossed. Likewise, it can also mean that actual changes in elevation are not detected when data points were recorded inside the same contour line. In the absence of an existing method to reconstruct accurate elevation data, we applied the same 9-point weighted average smoothing procedure we used in the speed data to calculate more realistic elevation data. Gradient was then calculated as the change in elevation divided by the horizontal distance between two points.

Paragraph \#10. The identification of relevant data was performed for each participant using official race results, GPS time-stamps and variations of speed (increase or decrease) indicating changes in position at the start and finish lines. Speed and gradient values were computed as a function of relative distance for each participant, where total individual distance represents 100\% of the distance completed for the $173-\mathrm{km}$, and $60 \%$ for the $103-\mathrm{km}$ event participants. Relative distance was used instead of the actual distance values because of the difference in total distances across participants. The dynamics of speed were determined relative to each participant's average speed over the entire event $(100 \%)$ in order to increase the relevance of inter-individual 
160

161

162

163

164

165

166

167

168

169

170

171

172

173

174

175

176

177

178

179

180

181

182

183

184

185

186

187

comparisons. The mean relative speed values corresponding to level (LEV; -2.5 to $2.5 \%$ gradient), uphill (UH; 2.5 to $100 \%$ gradient), and downhill (DH; -100 to $-2.5 \%$ gradient) were computed in sections of $5 \%$ of the total distance completed, which ensured a sufficient amount of data points in each section in the three gradient categories. The minimal amount of data points within one section occurred at $25 \%$ of total distance in the level gradient category ( 64 observations for one participant, which corresponded to $2.76 \mathrm{~km}$ for the participant), for an average number of observations in each gradient category of $360 \pm 98$ (LEV), $439 \pm 60$ (UH) and $386 \pm 67$ (DH).

Paragraph \#11. We initially assessed the normality (Kolmogorov-Smirnov test) and homogeneity of variances (Fisher's F test) of the 173-km and 103-km participants for LEV, UH, DH, and overall relative speeds. We then used independent t-tests to evaluate whether differences in relative speed existed between the $173-\mathrm{km}$ and $103-\mathrm{km}$ participants in the same categories. Due to varying lengths of data sets (173-km and 103-km distances), we investigated the dynamics of speed within each gradient category using a one-way ANOVA on ranks (Kruskal-Wallis test) and a pairwise multiple comparison (Dunn's method) when required, to determine and locate potentially significant differences between sections of relative distance.

Paragraph \#12. The relationship between the level of performance (individual average speed) and the variability of speed (coefficient of variation of speed), the magnitude of speed loss (slope of the linear regression of speed over the entire event) and the total time stopped (assumed to correspond to resting, eating, clothing and gear change, toilet, other), were assessed using correlations. Assumptions of normality were first tested using a Shapiro-Wilk test, and Pearson's product-moment correlation was used to calculate each relationship.

Paragraph \#13. All statistical analyses were performed using the computing program and its associated packages R (R Core Team 2015). The level of significance was set at $p<0.05$.

\section{Results}

Paragraph \#14. The datasets from four participants were not included in the analysis, due to either not completing the event, or discrepancies between official results and GPS data (difference in finish time greater than 5\%). A total of 15 GPS datasets were used for analysis (see Figure 1): ten participants completed the $173-\mathrm{km}$ distance averaging $32.9 \pm 3.6 \mathrm{~h}$ (range $26.5-36.3 \mathrm{~h}$ ), with a 
188 mean total distance of $173.0 \pm 4.0 \mathrm{~km}\left(5.68 \pm 1.51 \mathrm{~km} \cdot \mathrm{h}^{-1}\right)$, and five participants completed the 189 103-km distance averaging $18.9 \pm 2.3 \mathrm{~h}($ range $15.6-21.1 \mathrm{~h})$ with a mean total distance of 101.9 $190 \pm 2.3 \mathrm{~km}\left(5.91 \pm 1.48 \mathrm{~km} \cdot \mathrm{h}^{-1}\right)$. Weather conditions on race day were dry (no precipitations), the 191 temperatures ranged $14.9-22.8^{\circ} \mathrm{C}$ at the start point, $10.2-29.1{ }^{\circ} \mathrm{C}$ at the $103-\mathrm{km}$ checkpoint, and $192 \quad 11.4-26.8^{\circ} \mathrm{C}$ at the finish line, respectively.
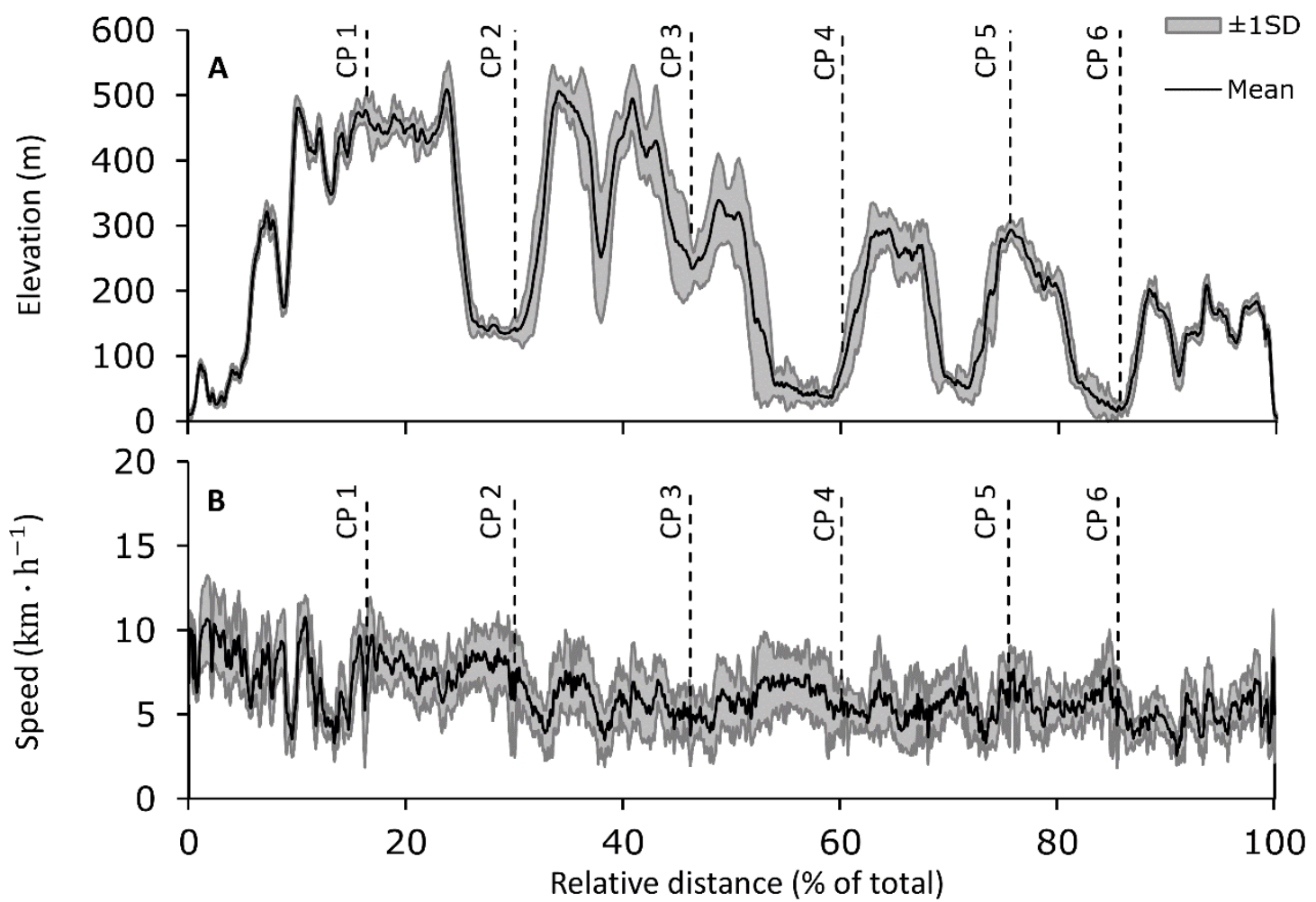

193

Figure 1. Elevation and speed. (A) Mean (SD) group DEM data from geographical positions. (B) 195 Mean $( \pm \mathrm{SD})$ group calculated speed. Abbreviation: CP are official race checkpoints.

197 Paragraph \#15. The initial testing for normality and homogeneity of variances did not reveal any 198 significant differences between 173-km and 103-km participants. There were no significant differences in means in the relative speed of the $173-\mathrm{km}$ and $103-\mathrm{km}$ participants for all categories of gradients (overall: $F=-1.38, p=0.17$;EV: $F=-0.99, p=0.32 ; U H:-1.37, p=0.18 ; \mathrm{DH}: F=$ 201 $-0.27, \mathrm{p}=0.79)$. Therefore, all remaining analysis include the $173-\mathrm{km}$ and $103-\mathrm{km}$ participants.

Paragraph \#16. Positive pacing was observed in all participants and in all gradient categories, except in one participant in negative gradients. The mean decrease in speed was $-4.35 \pm 3.0$ 
$204 \mathrm{~km} \cdot \mathrm{h}^{-1}(\mathrm{LEV}),-2.26 \pm 1.4 \mathrm{~km} \cdot \mathrm{h}^{-1}(\mathrm{UH})$, and $-4.36 \pm 2.2 \mathrm{~km} \cdot \mathrm{h}^{-1}(\mathrm{DH})$. Relative speed was 205 the most variable in LEV (coefficient of variation: 0.32 ) and reached a minimum at $50 \%$ of total 206 distance (significantly lower than all observations between 5\% and 40\%). The relative speed 207 increased from $50 \%$ to $60 \%, 85 \%, 90 \%$, and $100 \%$, and did not significantly decrease between any 208 sections before $50 \%$ and 100\% (Figure 2.A). Relative speed was the least variable in UH 209 (coefficient of variation: 0.22) and reached a minimum at 95\% of total distance. Relative speed 210 increased from $35 \%, 40 \%, 45 \%$ and $50 \%$ to $60 \%$. Relative speed increased from $95 \%$ to $100 \%$, 211 which was not different to any other observation (Figure 2.B). Relative speed in DH reached a 212 minimum at $95 \%$ of total distance (coefficient of variation: 0.26 ). The relative speed at $20 \%$ 213 increased compared to $15 \%$, but no other significant increase was observed. The relative speed at $21460 \%$ was not significantly different to any other observation, and the relative speed at $100 \%$ was 215 significantly lower than 5\%, 10\%, 15\%, 20\%, 30\% and 35\% (Figure 2.C). 


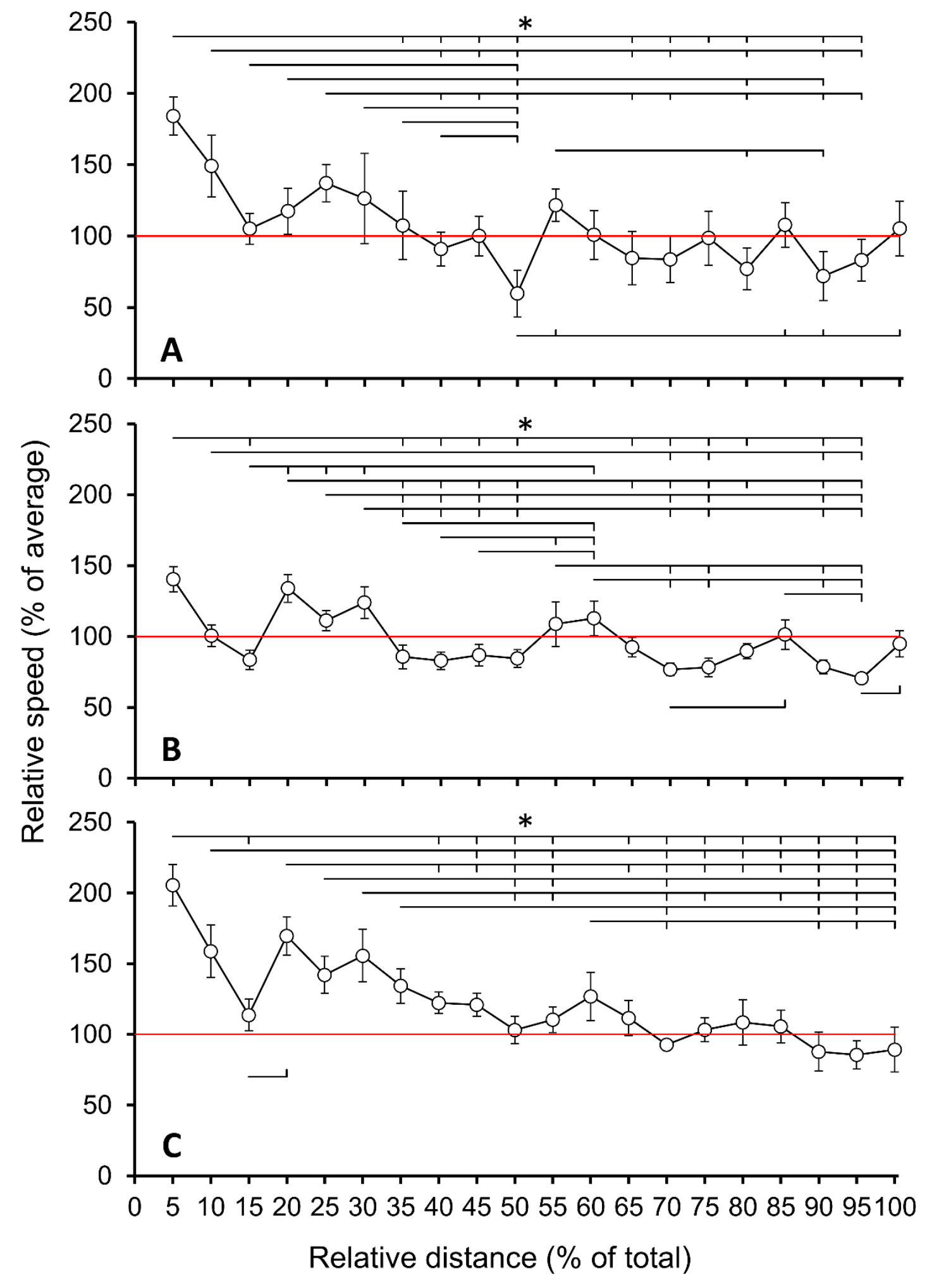


217 Figure 2. Dynamics of speed. Group level (Panel A), uphill (Panel B), and downhill (Panel C)

218 speed relative to average, respectively. Symbols * and brackets are used to denote and locate

219 significant differences $(\mathrm{p}<0.05)$.

220

221 Paragraph \#17. There were no significant correlations between overall performance and variables

222 of pacing (Table 1). The dynamics of total time stopped as a function of relative distance are 223 presented in Figure 3.

224

225 Table 1. Correlation between race performance and pacing characteristics.

\begin{tabular}{l|c|c|c|c|c|c}
\multicolumn{1}{c}{ All participants } & \multicolumn{2}{c|}{ 103-km } & \multicolumn{2}{c}{ 173-km } \\
& $r$ & $P$ & $r$ & $p$ & $r$ & $p$ \\
\hline Speed variability & -0.23 & 0.42 & -0.24 & 0.70 & -0.25 & 0.48 \\
\hline Speed loss & -0.24 & 0.39 & -0.43 & 0.47 & 0.00 & 1.00 \\
\hline Total time stopped & -0.35 & 0.21 & -0.15 & 0.81 & -0.45 & 0.20
\end{tabular}

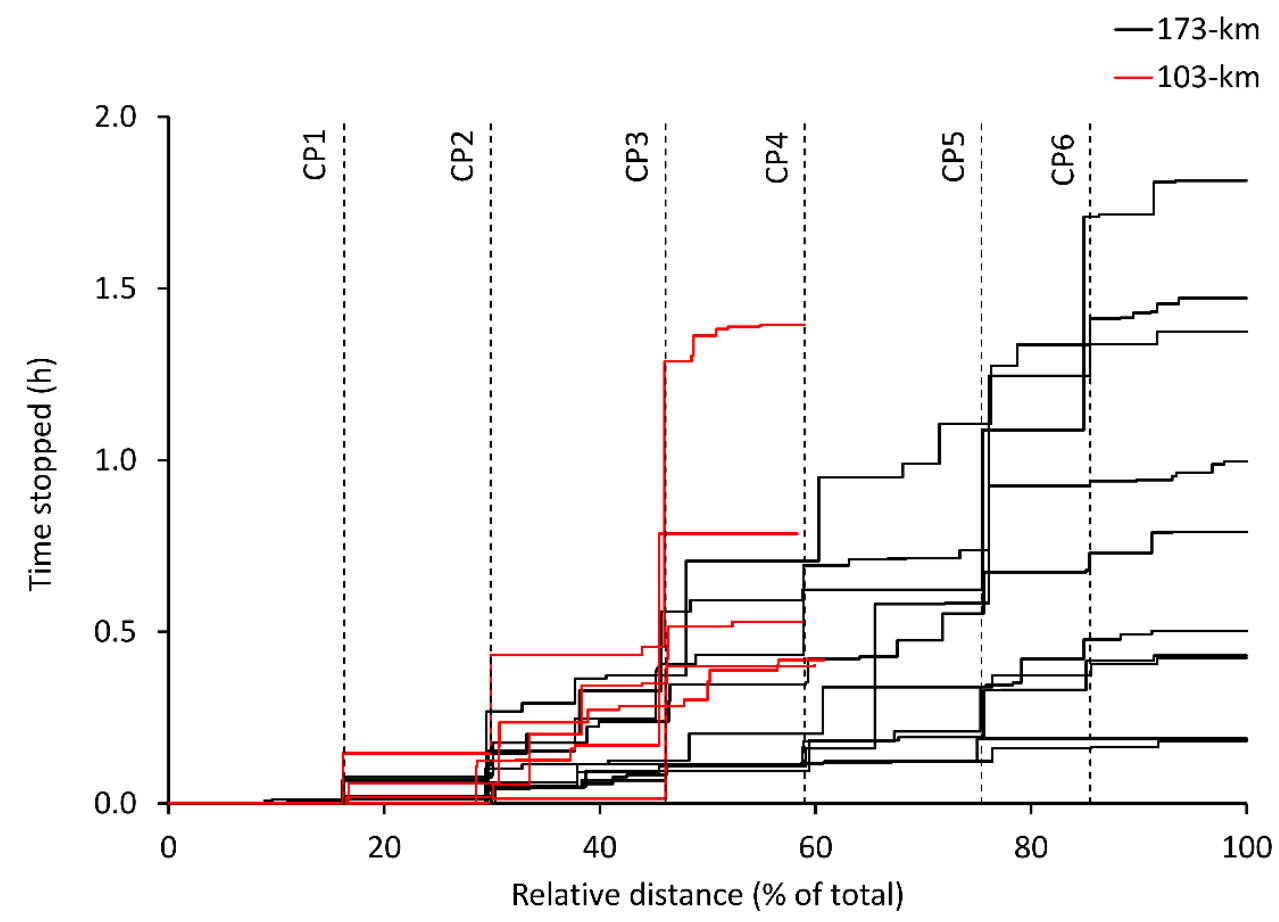


227 Figure 3. Total time stopped. Time stopped as a function of relative distance in all study

228 participants, using the cumulated durations associated with speed $<1 \mathrm{~km} \cdot \mathrm{h}^{-1}$.

\section{Discussion}

231

232

233

234

235

236

237

238

239

240

241

242

243

244

245

246

247

248

249

250

251

252

253

254

255

Paragraph \#18. In this study, we collected and reported the longest systematic description of pacing of runners in a long, hilly UM running event, using a method that created no disturbances to normal running event situations.

Paragraph \#19. The primary finding of this study was that positive pacing (overall decrease in speed) was used in all gradient categories, with three direct observations. Firstly, the variability of speed was higher in LEV, and, unlike in $\mathrm{UH}$ and $\mathrm{DH}$, speed loss was the greatest in the first half of the event. These observations are characteristic of a subset of the three main types of pacing referred to as parabolic or mixed pacing, with a positive pacing strategy during the first half of the event, and more evenly pace for the remainder of the event. Secondly, a speed reserve (increase in the last stage of an event) was measured in LEV and UH. Thirdly, the decrease in speed was greater in $\mathrm{DH}$ and LEV compared to $\mathrm{UH}$, and the decrease in relative speed continued until the last section in DH only. Together, these findings clearly indicate that despite slowing down overall, LEV, UH and DH were paced differentially. A secondary finding of this study was that neither the characteristics of relative speed (no significant differences in normality, variances or means), nor the characteristics of pacing (no differences in speed loss, variability of speed or total time stopped) were different in the $103-\mathrm{km}$ and $173-\mathrm{km}$ distances. This allowed us to compare the two groups and increase the relevance of our findings, but this finding also introduces the possibility that the additional $\sim 70 \mathrm{~km}$ did not significantly alter pacing in an ultramarathon performed in hilly terrain.

Paragraph \#20. A recent study from our group also reported parabolic pacing during a $105 \mathrm{~km}$ mountain UM, characterised by a decrease of speed at all gradient categories in the later stages of the event (70-90\% of total event duration), and by a final increase in speed in the last $10 \%$ of the event (Kerhervé et al. 2015). The presence of an increase in speed in the last $10 \%$ section of the event was discussed as an indicator of conservative pacing strategies in anticipation of upcoming topographic difficulties, and the use of a speed reserve (Millet 2011) when the last topographic difficulty was passed. In the current study, both the longer distance and smaller elevation gain and 
256 loss could potentially explain the absence of a speed reserve in DH. Other research have noted the

257 determinant role of elevation gain and loss in the type and magnitude of fatigue on neuromuscular

258 (Martin et al. 2010; Millet et al. 2011b) and biomechanical alterations (Millet et al. 2011a; Morin

259 et al. 2011b). Alternatively, this finding could also potentially highlight the selective effects of

260 fatigue as a function of gradient category, specifically to limit the effect of the transmission of

261 force or vibrations to the musculo-skeletal systems, as previously indicated by the alteration of

262 running economy in LEV and DH gradient categories, but not in UH (Morin et al. 2011b; Vernillo

263 et al. 2015).

264 Paragraph \#21. The absence of significant correlations between overall performance and 265 indicators of speed variability, speed loss and total time stopped are novel. While speed loss and 266 total time stopped were found to be significantly correlated with performance in a mountain UM 267 (Kerhervé et al. 2015), speed loss and speed variability were found to be significantly correlated 268 with performance obtained in marathon (Ely et al. 2008; Haney \& Mercer 2011) and UM 269 (Hoffmann 2014; Lambert et al. 2004) running. Therefore, additional research is required to 270 determine if specific pacing characteristics are useful predictors of performance in long UM, and 271 whether other variables currently not measured could be better predictors of pacing, including but 272 variables of gait kinematics (stride frequency, stride length, ground contact and aerial times) or 273 gait biomechanics (leg and vertical stiffness), which are known to be influenced by fatigue and 274 gradient (Morin et al. 2011a; Morin et al. 2011b; Vernillo et al. 2016; Vernillo et al. 2015; Vernillo 275 et al. 2014). Additionally, it has been proposed that the correlation between the level of 276 performance and a more even pace is due to learning (Foster et al. 1994; Green et al. 2010), and 277 that the previous practice of a specific distance produces more even pacing (Ansley et al. 2004; 278 Green et al. 2010). However, ultra-endurance events require longer recovery periods than shorter 279 events and hence, the opportunities to practice a specific distance are relatively less than for shorter 280 events, which could partly explain both the relative lack of data on longer events (Abbiss \& Laursen 2008), and the lack of a significant correlation of these variables in the current study.

282 Paragraph \#22. There were three direct limitations to this study. First, the findings related to the 283 variations of speed within sections of relative distance used binary results (different or not), but do not provide an estimation of the magnitude of differences. A simple level of analysis is not only acceptable, but also warranted, for the type of data used in this study. Future studies are required 286 to describe more accurately the direction and magnitude of changes. Second, the inclusion of 
287 participants from both the 103-km and 173-km distances could have implications for the results,

288 due to any differences in a priori pacing strategies across the two distances, or information relative

289 to the decision to stop for the 103-km group. For example, the five participants who had entered

290 the 173-km event and stopped at the 103-km distance might have done so due to an inappropriate

291 pacing strategy, or to a deliberate strategy. Irrespective, we reported the outcomes of pacing, which

292 incorporate both inappropriate and deliberate strategies, and provided evidence that the general

293 patterns of pacing did not differ across groups. Third, there are potential carry-over effects at

294 transitions between types of gradients during running (Townshend et al. 2009). However, the

295 spatial and temporal resolutions permitted by non-differential GPS do not currently allow

296 sufficient accuracy for this level of analysis during UM events, and future studies using alternative

297 methodologies are necessary to investigate these effects.

298 Conclusion

299 Paragraph \#23. In conclusion, in this study we determined the dynamics and characteristics of 300 speed of UM runners in an actual event, which provides a basis for future studies of ultra-long 301 duration exercise. While the speed of all participants decreased as a function of distance over the

302

303

304

305

306

307

308

309

310

311 The authors would like to thank Dr. Andrew Townshend for his valuable comments in the

312 conception of the study. 


\section{Figure legends}

314 Figure 1. Elevation and speed. (A) Mean (SD) group DEM data from geographical positions.

315 (B) Mean $( \pm \mathrm{SD})$ group calculated speed. Abbreviation: $\mathrm{CP}$ are official race checkpoints.

316 Figure 2. Dynamics of speed. Group level (Panel A), uphill (Panel B), and downhill (Panel C)

317 speed relative to average, respectively. Symbols * and brackets are used to denote and locate 318 significant differences $(\mathrm{p}<0.05)$.

319 Figure 3. Total time stopped. Time stopped as a function of relative distance in all study

320 participants, using the cumulated durations associated with speed $<1 \mathrm{~km} \cdot \mathrm{h}^{-1}$. 
Abbiss CR, and Laursen PB. 2008. Describing and understanding pacing strategies during athletic competition. Sports medicine 38:239-252 10.2165/00007256-200838030-00004

Angus SD, and Waterhouse BJ. 2011. Pacing strategy from high-frequency field data: more evidence for neural regulation? Medicine and Science in Sports and Exercise 43:2405-2412. 10.1249/MSS.0b013e3182245367

Ansley L, Schabort E, St Clair Gibson A, Lambert MI, and Noakes TD. 2004. Regulation of pacing strategies during successive 4-km time trials. Medicine and Science in Sports and Exercise 36:1819-1825. 10.1249/01.MSS.0000142409.70181.9D

Defense Mapping Agency. 1990. Datums, ellipsoids, and grids reference systems (Technical Manual 8358.1). In: DMA, editor. Fairfax, VA: US DoD.

Ely MR, Martin DE, Cheuvront SN, and Montain SJ. 2008. Effect of ambient temperature on marathon pacing is dependent on runner ability. Medicine and Science in Sports and Exercise 40:16751680. 10.1249/MSS.0b013e3181788da9

Foster C, Schrager M, Snyder AC, and Thompson NN. 1994. Pacing strategy and athletic performance. Sports medicine 17:77-85. 10.2165/00007256-199417020-00001

Gimenez P, Kerhervé H, Messonnier LA, Féasson L, and Millet GY. 2013. Changes in the energy cost of running during a 24-h treadmill exercise. Medicine and Science in Sports and Exercise 45:18071813. 10.1249/MSS.0b013e318292c0ec

Green MJ, Sapp AL, Pritchett RC, and Bishop PA. 2010. Pacing accuracy in collegiate and recreational runners. European Journal of Applied Physiology 108:567-572. 10.1007/s00421-009-1257-5

Haney TAJ, and Mercer JA. 2011. A Description of Variability of Pacing in Marathon Distance Running. International Journal of Exercise Science 4:article 6.

Herbst L, Knechtle B, Lopez CL, Andonie JL, Fraire OS, Kohler G, Rüst CA, and Rosemann T. 2011. Pacing strategy and change in body composition during a Deca Iron triathlon. Chinese Journal of Physiology 54:255-263. 10.4077/CJP.2011.AMM115

Hoffmann MD. 2014. Pacing by winners of a 161-km mountain ultramarathon. International Journal of Sports Physiology and Performance 9:1054-1056. 10.1123/ijspp.2013-0556

Kerhervé HA, Millet GY, and Solomon C. 2015. The dynamics of speed selection and psychophysiological load during a mountain ultramarathon. PLoS One 10:e0145482. 10.1371/journal.pone.0145482

Knicker AJ, Renshaw I, Oldham AR, and Cairns SP. 2011. Interactive processes link the multiple symptoms of fatigue in sport competition. Sports medicine 41:307-328. 10.2165/11586070000000000-00000

Lambert MI, Dugas JP, Kirkman MC, Mokone GG, and Waldeck MR. 2004. Changes in running speeds in a $100 \mathrm{~km}$ ultra-marathon race. Journal of Sports Science and Medicine 3:167-173.

Martin V, Kerhervé H, Messonnier LA, Banfi J-C, Geyssant A, Bonnefoy R, Féasson L, and Millet GY. 2010. Central and peripheral contributions to neuromuscular fatigue induced by a 24-h treadmill run. Journal of Applied Physiology 108:000-000. 10.1152/japplphysiol.01202.2009

Millet GY. 2011. Can neuromuscular fatigue explain running strategies and performance in ultramarathons?: The flush model. Sports medicine 41:489-506. 10.2165/11588760-000000000-00000

Millet GY, Banfi J-C, Kerhervé H, Morin J-B, Vincent L, Estrade C, Geyssant A, and Féasson L. 2011a. Physiological and biological factors associated with a $24 \mathrm{~h}$ treadmill ultra-marathon performance. Scandinavian Journal of Medicine and Science in Sports 21:54-61. 10.1111/j.16000838.2009.01001.x

Millet GY, Tomazin K, Verges S, Vincent C, Bonnefoy R, Boisson R-C, Gergelé L, Féasson L, and Martin V. 2011b. Neuromuscular consequences of an extreme mountain ultra-marathon. PLoS One 6:e17059. 10.1371/journal.pone.0017059 
Morin J-B, Samozino P, and Millet GY. 2011a. Changes in running kinematics, kinetics, and spring-mass behavior over a 24-h run. Medicine and Science in Sports and Exercise 43:829-836. 10.1249/MSS.0b013e3181fec518

Morin J-B, Tomazin K, Edouard P, and Millet GY. 2011b. Changes in running mechanics and springmass behavior induced by a mountain ultra-marathon race. Journal of Biomechanics 44:11041107. 10.1016/j.jbiomech.2011.01.028

R Core Team. 2015. R: A Language and Environment for Statistical Computing. Vienna, Austria: R Foundation for Statistical Computing.

Townshend AD, Worringham CJ, and Stewart IB. 2008. Assessment of speed and position during human locomotion using nondifferential GPS. Medicine and Science in Sports and Exercise 40:124-132. $10.1249 / \mathrm{mss} .0 \mathrm{~b} 013 \mathrm{e} 3181590 \mathrm{bc} 2$

Townshend AD, Worringham CJ, and Stewart IB. 2009. Spontaneous pacing during overground hill running. Medicine and Science in Sports and Exercise 42:160-169. 10.1249/MSS.0b013e3181af21e2

Vernillo G, Giandolini M, Edwards WB, Morin J-B, Samozino P, Horvais N, and Millet GY. 2016. Biomechanics and Physiology of Uphill and Downhill Running. Sports medicine. 10.1007/s40279-016-0605-y

Vernillo G, Savoldelli A, Zignoli A, Skafidas S, Fornasiero A, Torre AL, Bortolan L, Pellegrini B, and Schena F. 2015. Energy cost and kinematics of level, uphill and downhill running: fatigueinduced changes after a mountain ultramarathon. Journal of Sports Sciences 33:1998-2005. $10.1080 / 02640414.2015 .1022870$

Vernillo G, Savoldelli A, Zignoli A, Trabucchi P, Pellegrini B, Millet GP, and Schena F. 2014. Influence of the world's most challenging mountain ultra-marathon on energy cost and running mechanics. European Journal of Applied Physiology 114:929-939. 10.1007/s00421-014-2824-y

Vincenty T. 1975. Direct and inverse solutions of geodesics on the ellipsoid with application of nested equations. Survey Review XXIII 23:88-93. 10.1179/sre.1975.23.176.88 